\title{
A MATHEMATICAL SENSOR MODEL FOR INDOOR USE OF A MULTI-BEAM ROTATING 3D LIDAR
}

\author{
L.S. Tessema ${ }^{1,2, *}$, R. Jaeger ${ }^{2}$, U. Stilla ${ }^{1}$ \\ ${ }^{1}$ Photogrammetry and Remote Sensing, Technical University of Munich (TUM), Germany \\ - (lealem.tessema, stilla)@tum.de \\ ${ }^{2}$ Center for Applied Research, Karlsruhe University of Applied Sciences, \\ 76133 Karlsruhe, Germany - reiner.jaeger@hs-karlsruhe.de
}

KEY WORDS: Multi-beam LiDAR, Sensor model, Velodyne

\begin{abstract}
:
Our contribution presents a new perspective in the mathematical description of a rotating multi-beam LiDAR sensor, in a sense that we make use of projective geometry along with the "homogeneous general equation of the second degree" to parametrize scan lines. We describe the scan geometry of a typical multi-beam rotating 3D LiDAR by representing scan lines as pojective conics that represent a projective figure (a cone) in an embedding plane. This approach enables the parameterization of each scan line using a generic conic section equation. Most modeling approachs model spinning LiDAR sensors in terms of individual points sampled by a laser beam. On the contrary, we propose a model that provides a high-level geometric interpretation both for the environment and the laser scans. Possible application scenarios include exterior and interior calibration of multiple rotating multi-beam sensors, scan distortion correction and localization in planar maps.
\end{abstract}

\section{INTRODUCTION}

Light Detection and Ranging (LiDAR) technology has been rapidly advancing due to an increasing demand for 3D mapping in numerous applications such a s autonomous driving and service robotics. Multi-beam rotating LiDAR sensors are popular for use in autonomous mobile mapping and robotics. This is primarily due to their compact form factor and their capability to provide three-dimensional scans typically under 0.1 seconds. Of course, not mention their affordability compared to industry standard high-fiedlity 3D scanners. Velodyne sensors are one of the most popular LiDAR sensors of this type. In order to use such sensors in different applications such as Multi-sensor calibration, Indoor SLAM and change detection, a unified mathematical model is deemed necessary.

We first provide brief review of the mathematical mechanics behind projective geometry and conic sections and how conic sections relate to scan geometry. Then we will theoretically as well as empirically demonstrate that a 3D point measured with the scan mechanism described belongs to some conic section formed by the intersection of a cone at the origin and a plane in the scene. The empirical discussion is provided based on both a simulated data and real sensor data from the Velodyne VLP-16 LiDAR sensor.

\section{THEORETICAL BACKGROUND}

\subsection{LiDAR Sensor Models}

Accoring to Thrun et al. (2005) LiDAR sensor models generally belong to either of the tree categories: beam-based,scanbased,correlation-based and landmark-based. Different authors have proposed extended versions of LiDAR sensor models that belong to one or more of these categories. For example.Schaefer et al. (2017) extend the beam-based model

\footnotetext{
${ }^{*}$ Corresponding Author
}

through a physics motivated laser beam model that makes use of ray-tracing to build an exponential decay-rate map from raw sensor readings.

In contrast to beam-based approaches, scan-based models do not make use of ray tracing. Such models fundamentally build a likelihood map of the environment based on beam end points. This likelihood map is then later used to compute the likelihood of particular measurement.

As the name implies, correlation based models deal with finding the degree of match between a measurement and ground truth. The correlation between a sensor observation and a map is typically calculated using a map correlation function. Most matching based SLAM algorithms Grisettiy et al. 2005, Nüchter. 2009, Kohlbrecher et al. 2011; Hess et al., 2016) belong to this category.

Yu et al. (2014) proposed an inverse evidential model that converts raw Velodyne sensor data to an occupancy map under an assumption of a local ground plane. Their approach makes use of the dempster-shafer evidence theory to fuse 2D occupancy maps acquired from different locations.

Grant et al. (2018, 2013) Developed a SLAM solution for a multi-beam LiDAR based mapping that detects planes at the same time. Their plane detection algorithm makes use of the scan geometry in a similar fashion as presented in this paper. Particularly, the authors leverage on the concept of conicsections as products of intersection of planes and a virtual cone formed by a $360^{\circ}$ sweep of a LiDAR beam in space. In contrast, our paper presents the theoretical reasoning behind such assumptions in light of projective conics and provides a respective mathematical sensor model.

Landmark-based approaches do not explicitly deal with raw sensor readings. This class of models extract features such as 
interest points, geometric entities (e.g. lines and shapes) and semantic elements such as walls and doors that serve as reduced representations of the environment. The advantage in such models is that the dimensionality of the sensor data is significantly reduced while keeping track of the most salient features of the environment.

Our modeling approach in this paper belongs to this category. We assume a map of the environment, $\mathbf{m}$ containing planar features $m_{i}$ is given and we discuss the process of generating sensor observations from such a map.

Normally, features are localized in the environment with a position vector with respect to the sensor origin. For example, the location of a feature can be given by the vector $\left[r_{i}, b_{i}, c_{i}\right]^{T}$,where $r$ is the measured range, $b$ is bearing and $c$ is a parameter that identifies a unique feature, also known as a correspondence variable. We generalize this notion by considering unique planes given by the parameters $A, B, C$ and $D$ where $A x+B y+C z+D=0$ in the environment as features. It is obvious that one can infer $r_{i}$ and $b_{i}$ from these parameters.

2.1.1 Observation Equation Let a typical observation equation for a LiDAR sensor be stated as

$$
\mathbf{z}_{i}=h\left(\mathbf{x}_{i}\right)+\delta_{i},
$$

where $\mathbf{x}$ represent both the pose of the sensor and the landmarks, $\mathbf{z}$ is the acquired measurement, $h(\mathbf{x})$ is some function relating $\mathbf{x}$ with $\mathbf{z}$ and $\delta$ is a zero-mean Gaussian noise vector. The function $h(\mathbf{x})$ predicts expected observations based on the sensor pose $\mathbf{x}$ and a representation of the environment as a map m.

A single measurement of a typical multi-beam spinning LiDAR provides data of dimension $m \times n$ where $m$ is the number of laser rings and $n$ is the number of points sampled in a single sweep.

$$
\mathbf{z}=\left(\begin{array}{cccc}
r_{11} & r_{12} & \ldots & r_{1 n} \\
r_{21} & r_{22} & \ldots & r_{2 n} \\
\vdots & \vdots & \ddots & \vdots \\
r_{m 1} & r_{m 2} & \ldots & r_{m n}
\end{array}\right)
$$

Each $z_{i j}$ represents a single point measured from the $i^{\text {th }}$ laser beam at the $j^{t h}$ horizontal sample (bearing).

\subsection{Geometric Preliminaries}

We first present the analytic forms of quadric and planar surfaces in 3-dimensional Euclidean space $\mathbb{R}^{3}$ to be followed by a discussion of figures in the real projective plane $\mathbb{R P}^{2}$.

An arbitrary second order algebraic surface in $\mathbb{R}^{3}$ is given by the general equation of the second degree in $x, y$ and $z$. The equation of such a surface is

$$
\begin{aligned}
& F(x, y, z)= \\
& \begin{aligned}
A x^{2}+B y^{2}+C z^{2}+F x y+G y z+ \\
H x z+J x+K y+L z+M=0 .
\end{aligned}
\end{aligned}
$$

Let $\mathbf{Q}=\left(\begin{array}{ccc}A & 1 / 2 F & 1 / 2 H \\ 1 / 2 F & B & 1 / 2 G \\ 1 / 2 H & 1 / 2 G & C\end{array}\right), \mathbf{J}=\left(\begin{array}{c}J \\ K \\ L\end{array}\right)$ and $\mathbf{x}=\left(\begin{array}{l}x \\ y \\ z\end{array}\right)$
Then the matrix form of Equation 3 will be

$$
\mathbf{x}^{\mathrm{T}} \mathbf{Q} \mathbf{x}+\mathbf{J}^{\mathrm{T}} \mathbf{x}+M=0 .
$$

If the coefficients $J=K=L=M=0$, then we get the general second degree homogeneous equation (all terms with power 2) in the variables $x, y$ and $z$. This simplifies equation 4 into,

$$
\mathbf{x}^{\mathrm{T}} \mathbf{Q} \mathbf{x}=\mathbf{0}
$$

This is the general equation of an arbitrary elliptical cone centered at the origin. This cone is analogous to the virtual cone formed by a $360^{\circ}$ sweep of a single laser beam as will be discussed in Subsection 2.3

Similarly, an arbitrary plane in $\mathbb{R}^{3}$ is given by the general equation of the first degree in $x, y$ and $z$.

$$
A x+B y+C z+D=0
$$

Where $A, B$ and $C$ are not all zero and the normal vector of the plane is given by $\hat{\mathbf{n}}=A \hat{i}+B \hat{j}+C \hat{k}$ and $D=\hat{\mathbf{n}}^{T} P_{0}$ for any point $P_{0}$ that lies on the plane.

The theorem that conic sections are curves of intersection of a circular cone and a plane is well established and proven since 300 BC. Therefore, a formal proof will not be presented as we take this theorem for granted. The two-dimensional analogies of quadrics are conics given by the general equation

$$
A x^{2}+B x y+C y^{2}+F x+G y+H=0 .
$$

A summary of the classification of the general form of conic sections in $2 \mathrm{D}$ is given as follows.

\begin{tabular}{l|l} 
Conic type & Equation \\
\hline Parabola & $\mathrm{A} x^{2}+\mathrm{F} x+\mathrm{G} y=0$ \\
Circle & $x^{2}+y^{2}+F x+\mathrm{G} y+H=0$ \\
Ellipse & $\mathrm{A} x^{2}+\mathrm{C} y^{2}+\mathrm{F} x+\mathrm{G} y+H=0$ \\
Hyperbola & $\mathrm{A} x^{2}-\mathrm{C} y^{2}+\mathrm{F} x+\mathrm{G} y+H=0$
\end{tabular}

Table 1. Summary of general equations of conics

Alternatively, we may use the standard form of the equations as deemed necessary. For example the standard equation for a hyperbola formed as an intersection a right circular cone and a vertical plane is given in plane coordinates that satisfy

$$
\frac{y^{2}}{a^{2}}-\frac{x^{2}}{b^{2}}=1
$$

2.2.1 Projective Geometry In the so called Kleinian view, a geometry consists of a group of transformations acting on a space of points. If we for example take the group of orthogonal matrices, they are isometric Euclidean transformations in the space of $\mathbb{R}^{3}$, on the other hand, in the real projective space $\mathbb{R} \mathbb{P}^{n}$ they form a group of transformations with different mapping rules.

The real projective plane denoted as $\mathbb{R P}^{2}$ is a subset of the real projective space $\mathbb{R} \mathbb{P}^{n}$ which in turn is an abstract manifold. $\mathbb{R P}^{2}$ can simply be understood as the whole set of rays through the origin of $\mathbb{R}^{3}$. This set is topologically equivalent to points 
on the unit sphere that are the intersections of the rays and the surface of the unit sphere.

We now briefly revise the notions of a point, a line, a plane a conic in projective space as follows.

In $\mathbb{R P}^{2}$, each ray $\mathrm{X}$ (point on the unit sphere) is uniquely represented by the corresponding direction angles say given as $[a, b, c]$. These are the homogeneous coordinates that represent a line in $\mathbb{R}^{3}$ as a point in $\mathbb{R} \mathbb{P}^{2}$.

A line $\mathbf{l}$ in $\mathbb{R P}^{2}$ is represented by a plane through the origin of $\mathbb{R}^{3}$. Therefore the general form of equation of a line in homogeneous coordinates (homogeneous linear form) is given by the set of points with homogeneous coordinates $[x, y, z]^{\mathrm{T}}$ where

$$
a x+b y+c z=0 .
$$

The tuple $\mathbf{l}=(a, b, c)^{\mathrm{T}}$ uniquely represents a line in $\mathbb{R P}^{2}$. It follows that all projective points that lie on a line in $\mathbb{R P}^{2}$ should satisfy

$$
\mathrm{X}^{\mathrm{T}} \mathbf{l}=0 .
$$

We collectively refer to ellipses, parabolas and hyperbolas in projective space simply as projective conics. This generalization lends itself to the fact that ellipses, parabolas and hyperbolas are projective-congruent.

A projective conic in the real projective plane $\mathbb{R P}^{2}$ is a set of points $\mathrm{X}$ with homogeneous coordinates $[x, y, z]^{\mathrm{T}}$ that satisfy the homogeneous form of the general second degree equation

$$
A x^{2}+B x y+C y^{2}+F x z+G y z+H z^{2}=0 .
$$

In matrix form:

$$
\mathbf{X}^{\mathrm{T}} \mathbf{C X}=0
$$

Where,

$$
\mathbf{C}=\left(\begin{array}{ccc}
A & 1 / 2 B & 1 / 2 F \\
1 / 2 B & C & 1 / 2 G \\
1 / 2 F & 1 / 2 G & H
\end{array}\right)
$$

The matrix $\mathbf{C}$ uniquely represents a projective conic in $\mathbb{R P}^{2}$. For example, a hyperbola is given by the matrix

$$
\left(\begin{array}{ccc}
A & 0 & 1 / 2 F \\
0 & C & 1 / 2 G \\
1 / 2 F & 1 / 2 G & H
\end{array}\right) .
$$

The concept of an "embedding plane" is important and heavily used in our description. It let's us formalize the relationship between Euclidean figures in some plane $\pi$ with figures in $\mathbb{R P}^{2}$. A projective figure $\mathcal{F}_{p}$ in $\mathbb{R P}^{2}$ can be fully represented by a planar Euclidean figure in $\mathcal{F}_{e}$ embedded in $\pi$ along with a set of points known as ideal points that contain all projective points that lie on a plane through the origin parallel to $\pi$. These points are also referred to as vanishing points. Any arbitrary plane that does not pass through the origin of $\mathbb{R}^{3}$ can serve as an embedding plane.

The analogy between Equation 12 in $\mathbb{R P}^{2}$ and Equation 5 in $\mathbb{R}^{3}$ entails that a 3-dimensional conic surface in $\mathbb{R}^{3}$ is represented by a set of points $X$ with homogeneous coordinates that form a projective conic in an embedding plane $\pi$.
2.2.2 Projective Transformation Projective transformations are mappings from $\mathbb{R} \mathbb{P}^{2}$ to $\mathbb{R} \mathbb{P}^{2}$ given by

$$
t:[\mathrm{X}] \mapsto[\mathbf{H X}]
$$

where $\mathbf{H}$ is an invertible $3 \times 3$ matrix associated with the transformation function $t$. And $\mathrm{X}$ are points in homogeneous coordinates.

Projective transformations are important for the task at hand. If we consider a vertical plane as an embedding plane, some translation of a projective figure $\mathcal{F}_{p}$ in $\mathbb{R}^{3}$ (e.g. a cone) is equivalent to a projective transformation between two conic figures in $\mathbb{R} \mathbb{P}^{2}$. This implies that we can infer the translation in $\mathbb{R}^{3}$ from the projectivity of the two images of $\mathcal{F}$ on the same embedding plane $\pi$.

A transformation of homogeneous points $X$ on a projective conic $\mathbf{C}$ to points $\mathbf{X}^{\prime}$ that lie on other projective conic $\mathbf{C}^{\prime}$ under some transformation matrix $\mathbf{H}$ results in the transformation of $\mathbf{C}$ to $\mathbf{C}^{\prime}$ given by

$$
\mathbf{C}^{\prime}=\mathbf{H}^{-\mathrm{T}} \mathbf{C} \mathbf{H}^{-1}
$$

\subsection{Scan Mechanism}

If $\mathbb{R P}^{2}$ is the set of all lines passing through the origin in $\mathbb{R}^{3}$, laser rays spinning at an angle $\phi$ from the $x y$ plane about the $z$ axis of the sensor frame form a subset of $\mathbb{R P}^{2}$ which we call a projective figure $\mathcal{F}_{p}$. This figure is a degenerate quadric surface in $\mathbb{R}^{3}$, namely a double double napped cone.

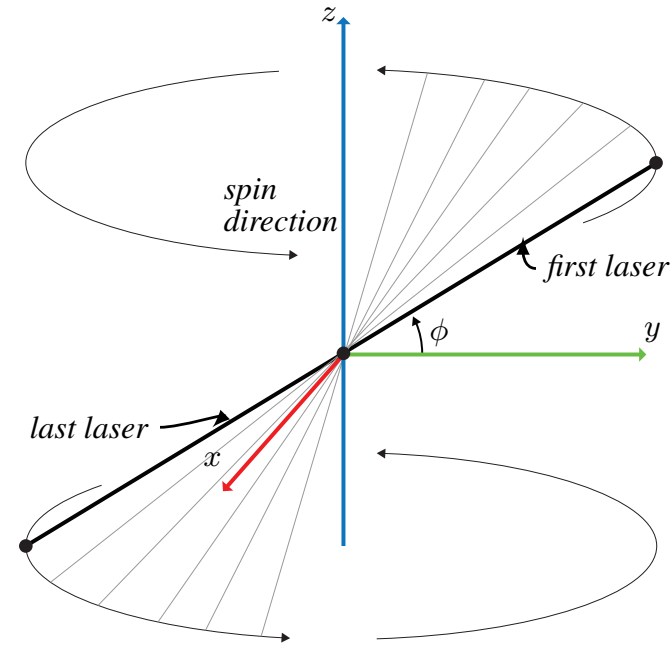

Figure 1. Scan mechanism for a typical spinning multi-beam LiDAR. We can consider the first and last laser beams to lie on a ray.

Another equivalent definition for a double-napped cone can be given as a surface generated by revolving a radius function $r(x)$ about the $z$ axis. Thus one can derive the equation for the surface using the equation of surface of revolution where $x^{2}+y^{2}=[r(z)]^{2}$. The equation for the surface of revolution formed by a laser beam at an inclination angle of $\phi$ is thus given by

$$
x^{2}+y^{2}=\frac{z^{2}}{\tan ^{2} \phi}
$$




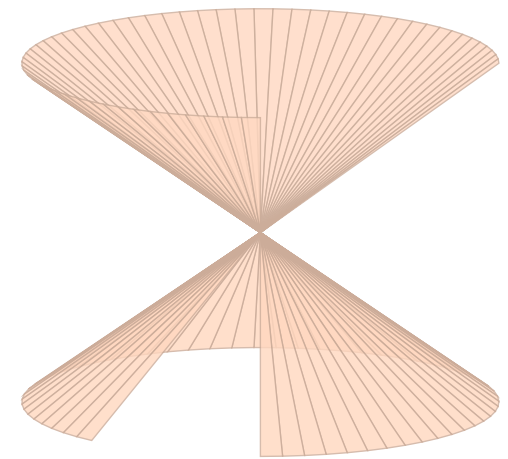

Figure 2. A double napped cone as a surface of revolution of a ray through the origin or $\mathbb{R}^{3}$.

\section{APPROACH}

As it is obvious by now, we intend to formulate a mathematical sensor model through the use of projective geometry by associating laser scans on a plane to a projective figure in $\mathbb{R P}^{2}$.

Just as an Euclidean figure $\mathcal{F}_{e}$ is defined to be a subset of $\mathbb{R}^{2}, \mathcal{F}_{p}$ is defined to be the subset of $\mathbb{R} \mathbb{P}^{2}$. The idea here is to represent the scan cone as a figure in a $2 \mathrm{D}$ embedding plane so that we infer some details regarding the figure without explicitly dealing with 3D Geometry. Now, the only restriction for an embedding plane being that it does not pass through the origin, we can assume arbitrary planes in a scene serve as embedding planes.

Considering vertical planes of an arbitrary rotation about the $z$-axis, the equation of such a plane is given by

$$
A x+B y+D=0
$$

Thus, the intersection of a right circular cone and a vertical plane forms a plane conic in the $x z$ or $x y$ planes. The equation of a conic in the $x z$ plane is given by the set of points satisfying

$$
\begin{aligned}
\{(x, y, z): & \\
& A x^{2}+B x z+C z^{2}+F x+G z+H=0, \\
& A x+B y+D=0\} .
\end{aligned}
$$

A plane given by $x-y=0$ results in a degenerate case where the embedding plane passes through the origin, and this defies the definition of an embedding plane. So we will not consider this situation as planes with this equation are not observable in reality.

\subsection{Assumptions}

As is the case that a model is a reduced representation of reality under assumptions, the following are assumptions made to confine the scope of our paper. They are not necessarily required for the modeling approach that follows.

\section{A. Stationary scans}

We assume all scans are acquired from a single point i.e. stationary scans. This is so to avoid distortion that is caused by motion. A single scan without motion conforms to the scan geometry discussed. Scan distortions as a result of motion can as well be explained with projective conics, but this is beyond the scope of this paper.

\section{B. Sensor orientation}

To simplify the algebraic equations and provide a clear view of the underlying process, we assume a sensor oriented horizontally i.e. parallel to the ground plane which in turn is assumed to be flat.

\section{Planar maps}

Indoor environments are typically composed of planes. We adopt the so called "Manhattan assumption" for simplification of the algebra. The proposed modeling approach does not depend on particular plane orientations. It can scale up to cope with other situations without loss of generality. Such maps could be available for example as GML basd maps (IndoorGML) or in a form of other BIM models. Keeping this in mind, elements of a map given as a collection of planes can be parametrized by Equation 6 In this case the coordinates are given with respect to the map origin, which is itself defined with the map.

\section{Deterministic approach}

We assume there are no distortions in the internal scan mechanism of the sensor. Most importantly, the inclination angles and the axis of spin are assumed to have their theoretical values. A distortion in these parameters can normally be detected through an intrinsic calibration procedures. Similarly, uncertainties in the geometry of the environment are not considered.

\subsection{Embedding a cone in a plane}

We begin by discussing the process of representing a right circular cone given by Equation 16 by a conic section in some embedding plane $\pi$. Let's assume the plane $\pi$ makes an angle $\theta$ with the positive $x$-axis and is perpendicular to the $x y$ plane.

Based on the formulations provided above, we can assume that every vertical plane in the scene is also an embedding plane for the projective figure. In order to describe the conic section just using two orthogonal Euclidean coordinates say $u, v$ that lie on $\pi$, we first rotate the $x y$ plane in $\mathbb{R}^{3}$ so that it is parallel to $\pi$. Let's call the new intermediate axes $x^{\prime}, y^{\prime}$ and $z^{\prime}$. We first rotate 90 degrees about the $x$-axis, this is due to the Manhattan assumption. Then we rotate along the $z$-axis so as to align with the axes of $\pi$. The corresponding rotation matrix is therefore given by

$$
\begin{aligned}
& \mathbf{H}_{r}=\left(\begin{array}{ccc}
1 & 0 & 0 \\
0 & 0 & -1 \\
0 & 1 & 0
\end{array}\right)\left(\begin{array}{ccc}
\cos \theta & -\sin \theta & 0 \\
\sin \theta & \cos \theta & 0 \\
0 & 0 & 1
\end{array}\right), \\
& \mathbf{H}_{r}=\left(\begin{array}{ccc}
\cos \theta & -\sin \theta & 0 \\
0 & 0 & -1 \\
\sin \theta & \cos \theta & 0
\end{array}\right)
\end{aligned}
$$

Once the $x^{\prime} y^{\prime}$ plane is parallel to $\pi$, we translate it through the new $z^{\prime}$ axis say by some distance $d$, so that the origin lies on $\pi$. Let's assume the final translated axes are $u, v$ and $w$ corresponding to $x, y$ and $z$. Since we are interested in the points of intersection of the plane $\pi$ and the projective cone, and as 


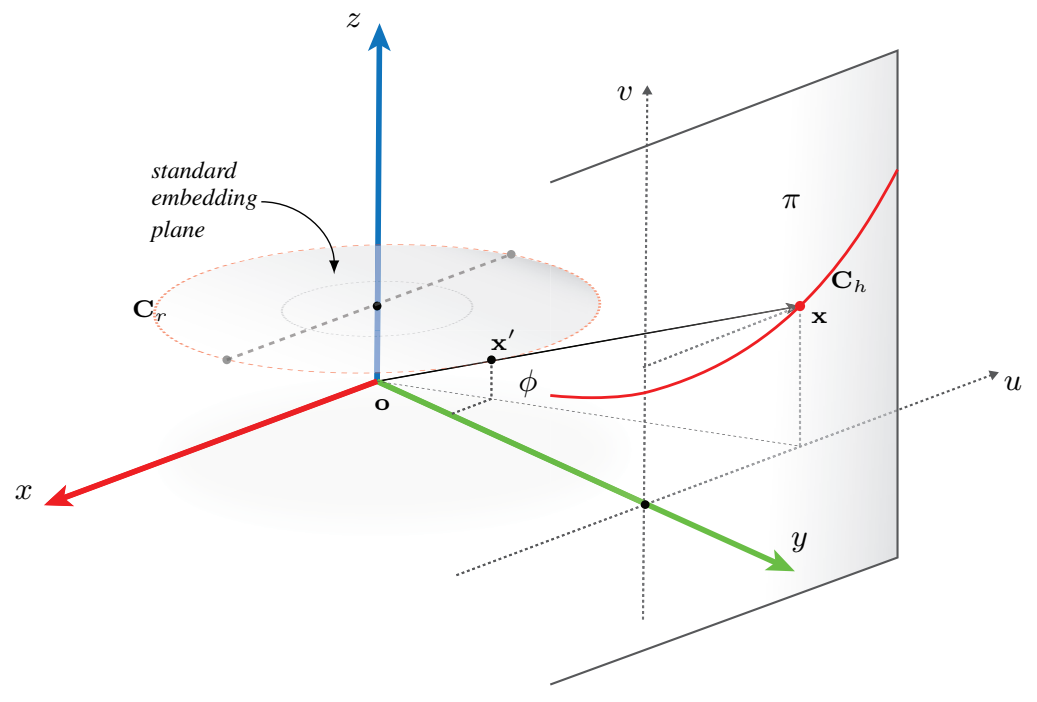

Figure 3. Projectivity between points on the standard embedding plane and points on a projective conic in an embedding plane $\pi$.

these points completely lie on a plane, we set $w=0$. The final transformation is given by

$$
\left(\begin{array}{l}
x \\
y \\
z
\end{array}\right)=\left(\begin{array}{ccc}
\cos \theta & -\sin \theta & 0 \\
0 & 0 & -1 \\
\sin \theta & \cos \theta & 0
\end{array}\right)\left(\begin{array}{l}
u \\
v \\
0
\end{array}\right)+\left(\begin{array}{c}
0 \\
-d \\
0
\end{array}\right) .
$$

Equation 20 transforms the coordinates of points on the embedding plane, $u, v$ and $w$ to $x, y$ and $z$ of $\mathbb{R}^{3}$ respectively. Accordingly the values for $x, y$ and $z$ can be formulated as

$$
\begin{aligned}
& x=u \cos \theta+v \sin \theta, \\
& y=-d, \\
& z=u \sin \theta+v \cos \theta .
\end{aligned}
$$

Substituting $x, y$ and $z$ in to Equation 16 and assuming $\theta=\pi / 2$ for simplicity, we get

$$
\frac{u^{2}}{d^{2} \tan ^{2} \phi}-\frac{v^{2}}{d^{2}}=1
$$

Since both of the denominators on the left hand side terms are constants, this is the standard form of the equation of a hyperbola given in Equation 8 This serves as a proof that, under the Manhattan assumption, the projective cone in $\mathbb{R} \mathbb{P}^{2}$ is represented as a hyperbola in all vertical planes of the scene.

\subsection{Projectivity between conics}

The use of homogeneous coordinates arises from the presence of a projectivity between points on different planes. We assume the plane $z=1$ to be the standard embedding plane and the process of representing figures in $\mathbb{R P}^{2}$ into the standard embedding plane is called standard embedding of $\mathbb{R P}^{2}$. We can make the following proposition based on this assumption.

\section{Proposition:}

There exists a unique a projectivity between the unit circle given by

$$
\begin{aligned}
\mathbf{C}_{u}=\left\{(x, y, z): x^{2}+y^{2}-1\right. & =0, \\
z-1 & =0\} .
\end{aligned}
$$

and the curve of intersection of the surface of revolution of a ray through the origin and an embedding plane given by $\mathrm{A} x+$ $\mathrm{B} y+\mathrm{D}=0$.

From projective geometry, we know that some set of lines passing through the origin can be represented by an Euclidean figure in an embedding plane $\pi$.

The standard embedding of the projective figure $\mathcal{F}_{p}$ represented as a subset of lines passing through the origin in $\mathbb{R}^{3}$ that satisfy Equation 16 leads to the following equation of a circle.

$$
\begin{aligned}
\mathbf{C}_{r}=\left\{(x, y, z): x^{2}+y^{2}-r\right. & =0, \\
z-1 & =0\} .
\end{aligned}
$$

Where $r=\tan (\pi / 2-\phi)$ and $\phi$ is the angle of inclination.

Let $\mathbf{C}_{h}=\left\{\mathbf{x}_{i}\right\}$ be set of points on the intersection of a revolving ray and an arbitrary plane with $\mathbf{x}_{i}=\left(x_{i}, y_{i}, z_{i}\right)^{\mathrm{T}}$ and $\mathbf{C}_{r}=\left\{\mathbf{x}_{i}^{\prime}\right\}$ with $\mathbf{x}_{i}^{\prime}=\left(x_{i}^{\prime}, y_{i}^{\prime}, 1\right)^{\mathrm{T}}$ be the corresponding sets of points satisfying Equation 24 The two sets of points are related by a $3 \times 3$ projective transformation matrix $\mathbf{H}$ where $\mathbf{x}_{i}=\mathbf{H} \mathbf{x}_{i}^{\prime}$. Given a minimum of four corresponding points, it would be trivial to show that the homography in this case is actually equal to the identity matrix. This informs us that the two sets of points are equivalent up to some scaling factor $\lambda$.

Now, the mapping from $\mathbf{C}_{r}$ to $\mathbf{C}_{h}$ is reduced to the estimation of $\lambda$ that brings each $\mathbf{x}_{i}^{\prime}$ to $\mathbf{x}_{i}$. For the sake of brevity, let's assume $\mathbf{C}_{h}$ to lie on a plane $y+\mathrm{D}=0$. We simply refer to this plane as $\pi$ here onwards. We derive the following trigonometric relationships that give us a pair of signed distances $r$ and $r^{\prime}$,

$$
\begin{aligned}
r & =\frac{\mathrm{D}}{\sin \theta_{i} \cos \phi}, \\
r^{\prime} & =\frac{y^{\prime}}{\sin \theta_{i} \cos \phi} .
\end{aligned}
$$


Where $\mathrm{D}$ is the perpendicular distance of $\pi$ from the origin and $\theta$ is the angle each vector in $\mathbf{C}_{r}$ makes with the horizontal $x$ axis given as the four-quadrant inverse tangent of the points $\left(x_{i}^{\prime}, y_{i}^{\prime}\right)$. From the property of perspectivity, we know that there exists some number $\lambda$ given by

$$
\lambda_{i}=\frac{r_{i}}{r_{i}^{\prime}}
$$

such that $\mathbf{x}_{i}=\lambda_{i} \mathbf{x}_{i}^{\prime}$. The interpretation of this result is, for a plane $\pi$, one can retrieve a unique representation of $\mathcal{F}_{p}$ in the plane by scaling every point $\mathbf{x}_{i}^{\prime}$ on $\mathbf{C}_{r}$ with $\lambda_{i}$. The figure formed this way will be a hyperbola.

From affine geometry, we know that all ellipses are affinecongruent. As a circle is a special case of an ellipse,it follows that every circle on the standard embedding plane can be represented by the unit circle and some factor $r$. Accordingly, the standard embedding of the set of projective figures $\mathcal{F}_{p}$ formed by $n$ rays spinning at different inclination angles $\phi$ results in a set of concentric circles which are affine-congruent to the unit circle on the same plane.

Now, there must exist a similarity transformation that maps $\mathbf{C}_{r}$ on to $\mathbf{C}_{u}$. A similarity transformation in $\mathbb{R}^{2}$ is given by a function $t: \mathbb{R}^{2} \mapsto \mathbb{R}^{2}$. We can represent the transformation using

$$
\mathbf{C}_{r}=\mathbf{A}^{-T} \mathbf{C}_{u} \mathbf{A}^{-1},
$$

where $\mathbf{A}$ is given by

$$
\left(\begin{array}{lll}
r & 0 & 0 \\
0 & r & 0 \\
0 & 0 & 1
\end{array}\right)
$$

Next, based on the discussion in the previous section, we compute the transformation that aligns the $x y$ plane to $\pi$. Equation 19 gives us the relative orientation between the two planes (i.e. $x y$ and $\pi$ ). Thus, a projective figure in the $x y$ plane $-\mathbf{C}_{r}$ is mapped to another projective figure $\mathbf{C}_{h}$ in $\pi$.

$$
\mathbf{C}_{h}=\mathbf{H}_{r} \mathbf{C}_{r} \mathbf{H}_{r}^{-1}
$$

For a plane $\pi$ with $\theta=90^{\circ}$, applying the corresponding matrix $\mathbf{R}$ in Equation 28 gives

$$
\mathbf{C}_{h}=\left(\begin{array}{ccc}
1 / r^{2} & 0 & 0 \\
0 & -1 / r^{2} & 0 \\
0 & 0 & 1
\end{array}\right)
$$

Equation 29 represents a hyperbola in $\mathbb{R P}^{2}$ given by

$$
x^{\prime 2}-y^{\prime 2}+r^{2} z^{\prime 2}=0 .
$$

The plane $x^{\prime} y^{\prime}$ is now parallel to $\pi$ but not at $\pi$. Based on the effect $\mathbf{R}$ has on the set of points $\mathbf{C}_{h}$ and $\mathbf{C}_{r}$ we conclude that it is a projective transformation in $\mathbb{R P}^{2}$ that maps points in plane $x y$ to corresponding points in a plane parallel to $\pi$. Since in $\mathbb{R P}^{2}$ the coordinates are homogeneous, we still need to apply a scaling (similarity) transformation to $\mathbf{C}_{h}$ to figure out its image in $\pi$. We then implement the translation from Equation 20 as a scaling (similarity) operation. This is carried out by the transformation matrix

$$
\mathbf{H}_{s}^{\prime}=\left(\begin{array}{ccc}
\mathrm{D} & 0 & 0 \\
0 & \mathrm{D} & 0 \\
0 & 0 & 1
\end{array}\right) .
$$

Where $\mathrm{D}$ is the perpendicular distance of $\pi$ from the origin.Accordingly, the projective conic in $\pi$ with a projectivity relation to the unit circle in the $x y$ plane is

$$
\mathbf{C}_{h}^{\prime}=\mathbf{H}_{s}^{\prime-T} \mathbf{C}_{h} \mathbf{H}_{s}^{\prime-1}
$$

Hence, we conclude that there is a projective transformation (homography) along with a scale factor that uniquely maps points on the unit circle in the standard embedding plane to a set of points satisfying Equation 22 on the plane $\mathrm{A} x+\mathrm{B} y+\mathrm{D}=0$. An interesting insight in this regard is, a rotation matrix in Euclidean $\mathbb{R}^{3}$ becomes a projective transformation matrix associated with a transformation function $t: \mathbb{R P}^{2} \mapsto \mathbb{R P}^{2}$.

\subsection{Rotating Multi-beam LiDAR Model}

We can formulate the sensor model as a projective mapping between $\mathbf{C}_{u}$ and $\mathbf{C}_{h}$. We do this by concatenating the series of transformations introduced in the previous section as

$$
\begin{aligned}
\mathbf{C}_{h} & =\mathbf{H}_{s}^{-\mathrm{T}}\left(\mathbf{H}_{r}^{-\mathrm{T}}\left(\mathbf{A}^{-\mathrm{T}} \mathbf{C}_{u} \mathbf{A}^{-1}\right) \mathbf{H}_{r}^{-1}\right) \mathbf{H}_{s}^{-1} \\
& =\mathbf{H}_{s}^{-\mathrm{T}}\left(\mathbf{H}_{r}^{-\mathrm{T}}\left(\mathbf{C}_{r}\right) \mathbf{H}_{r}^{-1}\right) \mathbf{H}_{s}^{-1}
\end{aligned}
$$

If we assume a generic projective transformation $\mathbf{H}$ that represents $\mathbf{H}_{s} \mathbf{H}_{r} \mathbf{A}$, equation 34 simplifies to

$$
\mathbf{C}_{h}=\mathbf{H}^{-\mathrm{T}} \mathbf{C}_{u} \mathbf{H}^{-1}
$$

which simply is the general rule for transformation of conic sections in homogeneous coordinates. Finally, we generalize Equation 33 for a landmark based map $\mathbf{m}$ given as a set of unique planes $m_{i}$. For each $m_{i}$, the corresponding observation model $h\left(m_{i}\right)$ for a vector of projective conics is given by

$$
\begin{aligned}
h\left(m_{i}\right) & =\left(\begin{array}{c}
\mathbf{C}_{h}^{1} \\
\vdots \\
\mathbf{C}_{h}^{n}
\end{array}\right) \\
& =\left(\mathbf{H}_{1}^{-\mathrm{T}} \cdots \mathbf{H}_{n}^{-\mathrm{T}}\right)\left(\begin{array}{ccc}
\mathbf{C}_{u} & & \\
& \ddots & \\
& & \mathbf{C}_{u}
\end{array}\right)\left(\begin{array}{c}
\mathbf{H}_{1}^{-1} \\
\vdots \\
\mathbf{H}_{n}^{-1}
\end{array}\right) .
\end{aligned}
$$

Where $\mathbf{H}_{i}$ is the corresponding projective transformation that maps points on the unit circle $\mathbf{C}_{u}$ to points on $\mathbf{C}_{h}$.

This gives the final result required. A model that represents the intrinsic geometric properties contained in the process of mapping planar environments with a multi-beam rotating LiDAR operating under the scan mechanism discussed herein.

Finally, we give an interesting insight regarding the property of the conic figures observed. Under the Manhattan assumption, each $\mathbf{C}_{h}^{i}$ will be a hyperbola and the eccentricity of the $i^{t h}$ hyperbola remains constant through out the planar map. On the other hand the directrix changes as a factor of the perpendicular distance to each plane in the map. 


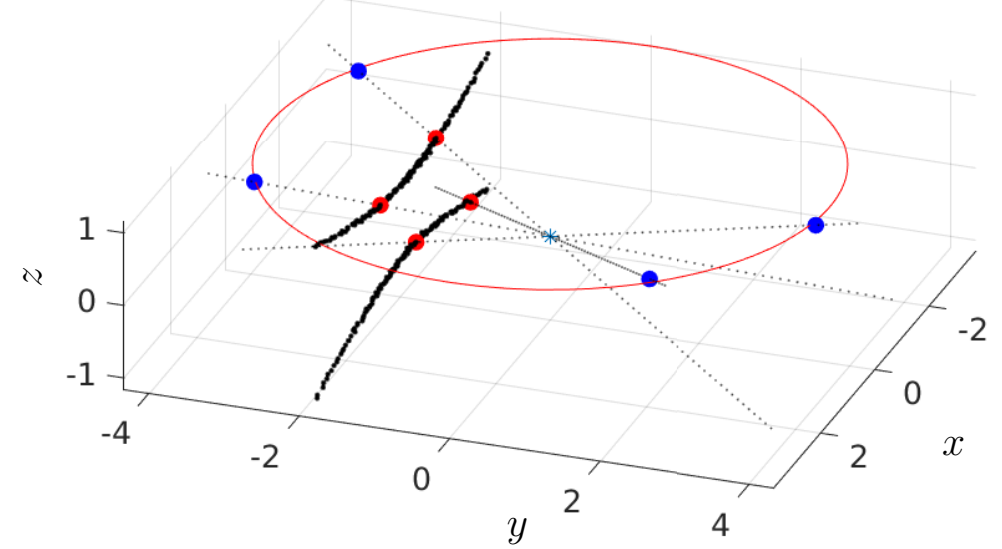

(a)

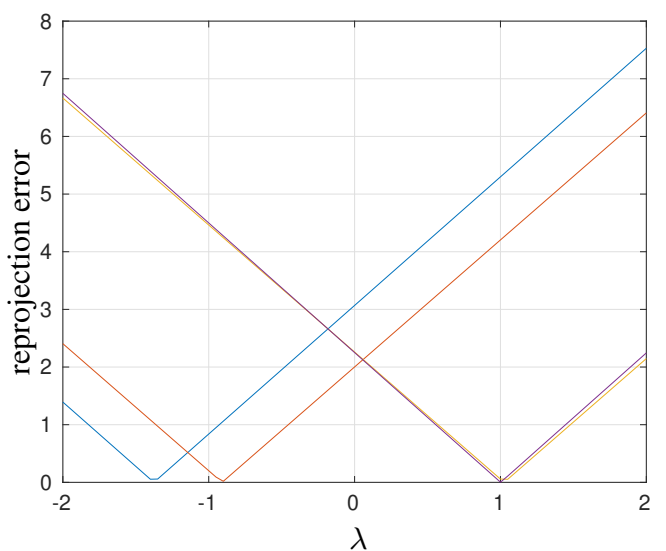

(b)

Figure 4. (a) shows sample points (blue) on $\mathbf{C}_{r}$ in a one-to-one projectivity with corresponding measured points (red) and (b) shows the reprojection error for different $\lambda_{i}$ values for each of the four sample points.

\section{EXPERIMENT}

In this section, we backup the theoretical discussion with empirical data. First we show that Equation 26 holds by taking a sample of four points from $\mathbf{C}_{r}$ and estimate the corresponding points on $\pi$. For this, we make use of scans at +15 and -15 degrees from a Velodyne VLP-16 LiDAR. From Figure 4, we see that there is indeed a unique scale factor $\lambda_{i}$ for each point that minimizes the norm of the distance $\left\|\mathbf{x}_{i}-\lambda_{i} \mathbf{x}_{\mathbf{i}}^{\prime}\right\|$ between the actual observations on $\pi$ and projections of points on $\mathbf{C}_{r}$. Under such transformation, it is also shown that a single projective figure (a circle) becomes a two 'bit' projective conic - a hyperbola.

Figure 5 shows the same LiDAR points along with the hyperbola that represents the set of observations on the $x z$ plane. The equation of the hyperbola is computed based on Equation 34 assuming the embedding plane is known before hand.

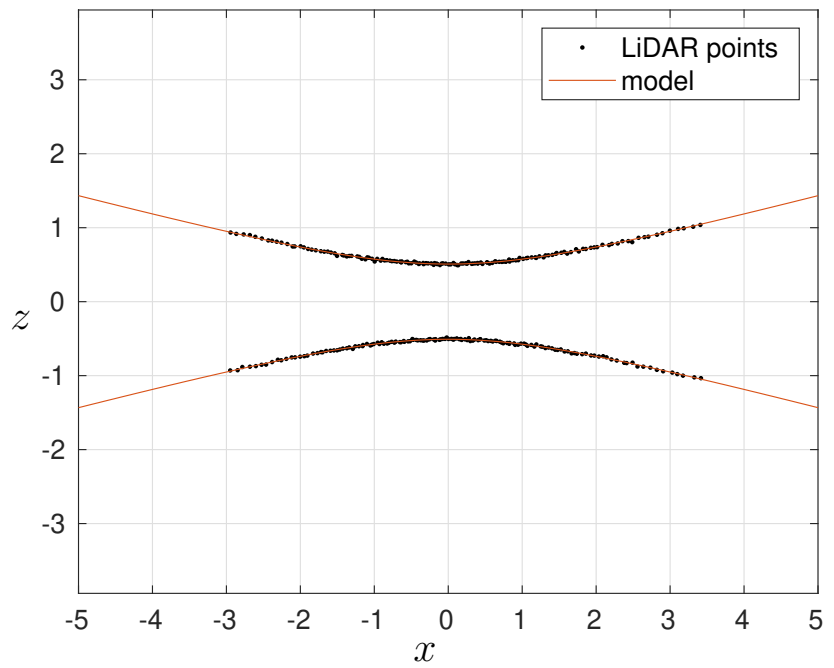

Figure 5. A hyperbola as a model representing scan points.

Figure 6a shows a complete scan of a typical indoor scene with the Velodyne VLP-16 laser scanner. A simple homogenization of all the coordinates of the points results in the corresponding representation in the real projective plane as shown in Figure $6 \mathrm{~b}$ The complete scan is represented as a set of 8 concentric circles in $\mathbb{R} \mathbb{P}^{2}$. This is due to the fact that the 16 laser beams of the sensor are arranged in such a way that half of them have positive inclination angles and the other half mirror the same inclination values but in the opposite direction.

Thus, the preposition made in Subsection 3.3 is shown to hold both in theory and practice. Observations of planes from a multi-beam LiDAR with an even number of laser beams $n$ with symmetric positive and negative inclination angles can be modeled by a projective mapping of the $n / 2$ concentric circles in the standard embedding plane on to planes in the map.

\section{CONCLUSION}

Typically, multi-beam rotating LiDAR sensors are constructed by positioning multiple range finders each positioned at a unique angle from the horizontal ( $x y$ ) plane and all spinning about the $z$ axis at the same rate. A single sweep forms an imaginary cone centered at the sensor origin. We exploited this geometry to describe scan lines as projective conics. In doing so, we made use of projective geometry and the concept of embedding planes to describe the sensor model.

We provided a discussion based on a special case where a Manhattan environment is assumed. But the same principles hold for non-Manhattan environments. The projective conics that appear in non-Manhattan, but still plane dominated environments in addition to hyperbolas will be ellipses and parabolas. Although, the approach adopted here is focused on modeling sensors operating in planar environments, the same concept can be expanded to a general case of non-planar surfaces through an approximation by a tangent plane at each scan point or groups of points.

In future works, we intend to investigate application scenarios that benefit from the use of our modeling approach. Possible application scenarios include calibration, localization in planar maps such as IndoorGML and plane detection in indoor scenes.

\section{ACKNOWLEDGEMENTS}

This work is financially supported by the Baden-Württemberg Minstry of Science, Research and Art. 


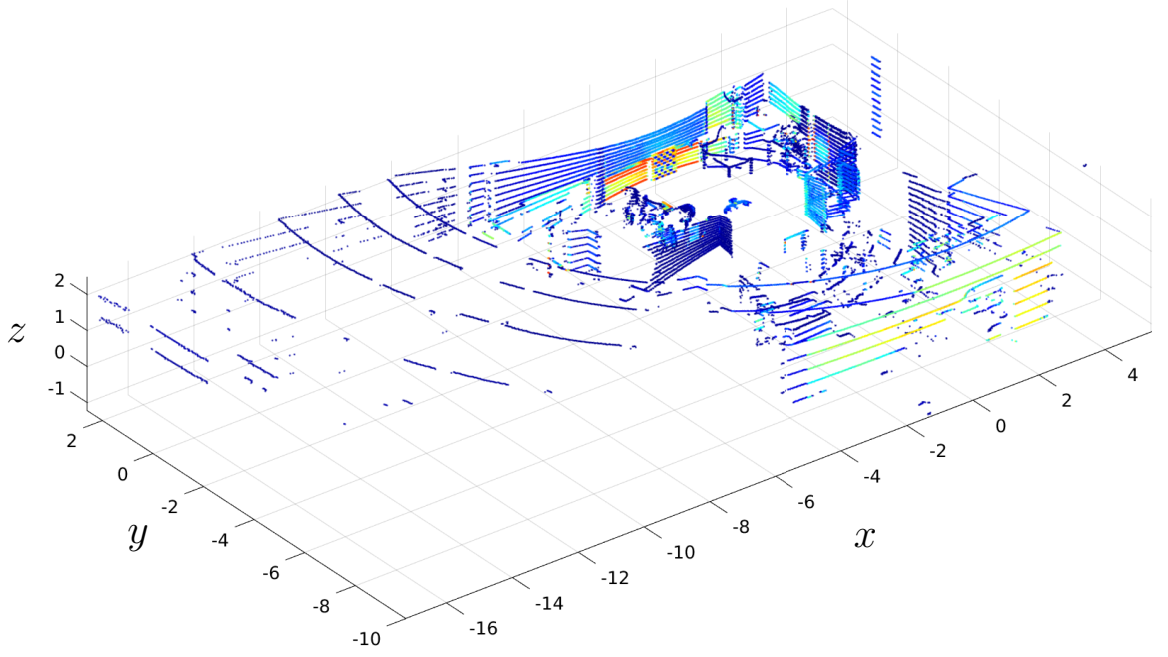

(a)

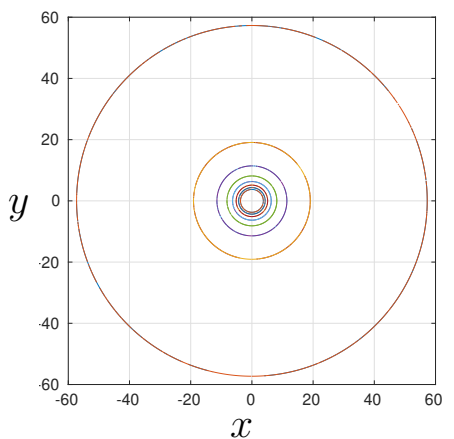

(b)

Figure 6. Real point cloud data from the Velodyne VLP-16 laser scanner in $\mathbb{R}^{3}$ (a) and in $\mathbb{R} \mathbb{P}^{2}$ (b).

\section{REFERENCE}

Grant, W.S., Voorhies, R.C., Itti, L., 2013. Finding planes in LiDAR point clouds for real-time registration. 2013 IEEE/RSJ International Conference on Intelligent Robots and Systems, IEEE, Tokyo, 4347-4354.

Grant, W.S., Voorhies, R.C., Itti, L., 2018. Efficient Velodyne SLAM with point and plane features. Autonomous Robots. doi.org/10.1007/s10514-018-9794-6.

Grisettiy, G., Stachniss, C., Burgard, W., 2005. Improving Gridbased SLAM with Rao-Blackwellized Particle Filters by Adaptive Proposals and Selective Resampling. Proceedings of the 2005 IEEE International Conference on Robotics and Automation, IEEE, Barcelona, Spain, 2432-2437.

Hess, W., Kohler, D., Rapp, H., Andor, D., 2016. Realtime loop closure in 2d LIDAR SLAM. 2016 IEEE International Conference on Robotics and Automation (ICRA), IEEE, Stockholm, Sweden, 1271-1278.

Kohlbrecher, S., von Stryk, O., Meyer, J., Klingauf, U., 2011. A flexible and scalable SLAM system with full 3d motion estimation. 2011 IEEE International Symposium on Safety, Security, and Rescue Robotics, IEEE, Kyoto, Japan,155-160.

Nüchter, A., 2009. 3D Robotic Mapping: the Simultaneous Localization and Mapping Problem with Six Degrees of Freedo$m$. 52, Springer.

Schaefer, A., Luft, L., Burgard, W., 2017. An Analytical Lidar Sensor Model Based on Ray Path Information. IEEE Robotics and Automation Letters, 2(3), 1405-1412.

Thrun, S., Burgard, W., Fox, D., 2005. Probabilistic Robotics. mit.

Yu, C., Cherfaoui, V., Bonnifait, P., 2014. An evidential sensor model for Velodyne scan grids. 2014 13th International Conference on Control Automation Robotics \& Vision (ICARCV),IEEE, Singapore, 583-588. 